\title{
Association analysis of LDLR gene polymorphisms with susceptibility to sudden deafness
}

\author{
Long Cui ${ }^{1,2}$, Wenjun Xin ${ }^{1}$, Jiawen Chen ${ }^{1}$, Weijun Ma1, Panhong Dang ${ }^{1}$, Maoli Duan ${ }^{1,3}$ and Xiaotong Zhang ${ }^{1 *}$ \\ ${ }^{1}$ Department of Otorhinolaryngology-Head and Neck Surgery, The Second Affiliated Hospital of Xi'an Jiaotong University, Xi'an, Shaanxi (710004), PR China \\ ${ }^{2}$ Department of Otolaryngology, Southern University of Science and Technology Hospital, Shenzhen, Guangdong (518055), PR China \\ ${ }^{3}$ Department of Otolaryngology Head and Neck Surgery, Karolinska University Hospital, 17176 Stockholm, Sweden
}

\begin{abstract}
In order to explore the association of 3 SNPs of $L D L R$ gene with susceptibility to sudden deafness (SD), serum lipid parameters were compared in 139 SD patients and 139 healthy individuals. Genotyping of the 3 SNPs, allele and genotype frequencies were performed and compared. The association between allele frequency of 3 SNPs and susceptibility to SD in different models were analysed. Serum lipid parameters were compared in patients with different genotypes. The comparison of serum lipid levels showed higher triglycerides, total cholesterol, and low-density lipoprotein cholesterol levels in patients compared to the controls. The frequencies of rs5929 T allele, rs $2738464 \mathrm{G}$ allele and rs1433099 $\mathrm{T}$ allele in SD group were higher than control group. We observed a statistically significant association of $L D L R$ gene rs5929 $\mathrm{T}$ allele under dominant genetic model, rs2738464 $\mathrm{G}$ allele under additive and dominant genetic models, rs1433099 $\mathrm{T}$ allele under dominant genetic model with susceptibility to SD. There was no statistical difference in serum lipid levels of SD patients with different genotypes. Total cholesterol, triglycerides, and low-density lipoprotein cholesterol may be risk factors in the pathogenesis of SD. rs5929 T allele, rs2738464 G allele and rs1433099 T allele may be risk factors for SD.
\end{abstract}

\section{Introduction}

Sudden sensorineural hearing loss (SD) is defined as a hearing loss in at least three contiguous test frequencies of $30 \mathrm{~dB}$ occurring within 3 days [1] and is one of the most common acute diseases of the ENT. Even with positive and timely treatment, many patients remain affected with permanent hearing loss, tinnitus, and other sequela that cause great suffering. While the etiology and pathogenesis remain to be elucidated, current research has subsequently focused on exploring polymorphisms in genes that are important for normal microcirculation. Indeed, genetic susceptibility to SD occurs in individuals with mutations in genes critical for microcirculation. This subsequently affects the inner ear microcirculation which is related to the incidence of SD. Mutations in genes encoding for methylenetetrahydrofolate reductase, prothrombin, and platelet glycoprotein are found in patients with microcirculation disorder and thus are genes of interest in understanding the basis of $\mathrm{SD}$ [2].

Several pieces of evidence suggest that abnormal lipid metabolism increases the risk of sudden sensorineural hearing loss $[3,4]$, Polymorphisms localized in the exons of low-density lipoprotein receptor $(L D L R)$ gene may have a significant impact on lipid metabolism by changing in the expression or stability of $L D L R[5,6]$. There has been no report on of the association of $L D L R$ gene polymorphisms with susceptibility to SD. Thus, in our study we compared 3 SNPs gene polymorphisms of $L D L R$ gene of 139 cases of patients of SD with 139 normal hearing volunteers.

The incidence of SD is estimated at 5-20/10,000 individuals per year ${ }^{[4]}$, so it is necessary to unravel the origins of SD. Exploring the relationship between $L D L R$ polymorphisms and susceptibility to SD may help identify SD susceptible populations and provide new methods for the prevention and diagnosis of SD.

\section{Methods}

\section{Statement}

All methods in this paper were performed in accordance with the relevant guidelines and regulations of the Second Affiliated Hospital of Xi'an Jiaotong University.

Informed consent has been obtained from a parent and/or legal guardian, for research involving human participants under the age of 18 years (including donors of tissue samples).

Informed consent has been obtained from adults themselves for research involving human participants (including donors of tissue samples).

\section{Participants}

SD group: 139 patients with SD visited the Department of Otolaryngology of the Second Affiliated Hospital of Xi'an Jiaotong University, from March 2013 to May 2014, included 70 males and 69 females, aged 17 to 72 years, mean $45.1 \pm 14.2$ years. All cases in this study were taken within the first 7 days of SD onset and provided initial treatment. Patients were selected by their relevant medical history and clinical examination to exclude hypertension, diabetes, coronary heart

${ }^{*}$ Correspondence to: Xiaotong Zhang, Department of OtorhinolaryngologyHead and Neck Surgery, The Second Affiliated Hospital of Xian Jiaotong University, No. 157. West 5th Road, Xi'an City, Shaanxi Province, China, Tel: 86 298266 8888; E-mail: tracylizhang@189.cn

Key words: Sudden deafness (SD), Serum lipid, LDLR gene, Single nucleotide polymorphisms (SNP)

Received: April 07, 2020; Accepted: April 23, 2020; Published: April 27, 2020 
disease, kidney disease, cancer, familial hyperlipidemia, obesity, by pure tone threshold and imaging examination to exclude otitis media, acoustic neuroma. Control group: The 139 individuals included 66 males and 73 females, aged 16 to 80 years, mean $45.3 \pm 14.3$ years, visited the Medical Center of the Second Affiliated Hospital of Xi'an Jiaotong University during the same time without hypertension, diabetes, coronary heart disease, kidney disease, cancer, familial hyperlipidemia, obesity and indicated no signs of ear disease (pure tone threshold to determine the normal hearing). All subjects were of Han nationality, Shaanxi province and unrelated; The age and sex characteristics were matched between the two groups. All samples were collected in line with "informed consent and informed choice" principle of medical ethics. This study was approved by the Second Affiliated Hospital of Xi'an Jiaotong University Ethics Committee.

\section{Reagents and methods}

All subjects had $5 \mathrm{ml}$ of venous blood drawn under fasting conditions (in the morning) without receiving any steroids treatment. $3 \mathrm{ml}$ of blood was placed in anti-coagulant tubes, and centrifugalized at $4^{\circ} \mathrm{C}, 3000 \mathrm{r} / \mathrm{min}$ for 15 minutes to prepare serum lipid levels test. Serum lipid parameters were tested on the Hitachi LABOSPECT008 automatic biochemical analyser: enzymatic method was used for detection of total cholesterol and triglycerides levels, ELISA method was used for detection of high-density lipoprotein cholesterol, low-density lipoprotein cholesterol levels, and projected immune turbidimetry was used for detection of apolipoprotein A1 and apolipoprotein B levels. $2 \mathrm{ml}$ of blood was placed in EDTA anticoagulant tubes, and frozen at $-80^{\circ} \mathrm{C}$ to prepare genomic DNA extractions. Whole blood DNA of small E.Z.N.A. quantity extraction kit (E.Z.N.A. Blood DNA kit II, the United States OMEGA) was used to extract 278 copies of sample DNA. First, $1 \mu \mathrm{l}$ of DNA sample was used to run on a $1 \%$ agarose gel through electrophoresis to estimate the concentration and inspect quality. Then DNA concentration was estimated according to the brightness of the image forming bands of ultraviolet imager. The samples were diluted to working concentration $(5-10 \mathrm{ng} / \mu \mathrm{l})$, iMLDR technology was applied to genotyping SNP loci.

\section{SNP Selection and Genotyping}

Three tSNPs were selected from the HapMap Project (International HapMap Consortium) using the following criteria: (1) a minor allele frequency $\geq 20 \%$ in the Han Chinese population and (2) heterozygosity $>10 \%$ in Chinese Han population. The three tSNPs included rs5929 at exon 13 in $L D L R$ gene, rs2738464 and rs1433099 at 3' untranslated region in $L D L R$ gene.

\section{Results}

Table 1 shows the comparison of serum lipid levels between the patients and the controls. The SD patients were with higher triglycerides, total cholesterol, and low-density lipoprotein cholesterol levels compared to the controls ( $\mathrm{P}<0.05 ; \mathrm{P}<0.01 ; \mathrm{P}<0.01$, respectively).

\section{LDLR gene Hardy-Weinberg equilibrium test}

Table 2 shows the genotype results of entire samples were imported directly into GeneMapper 4.1 software for analysis. 3 SNPs loci were in Hardy-Weinberg equilibrium $(\mathrm{P}>0.05)$, and this study showed that selected samples had group representation.

\section{Statistical analysis of 3 SNPs of $L D L R$ gene}

Table 2 shows the comparison of 3 SNPs of SD group and control group in allele frequency. In SD group, the frequency of $L D L R$ gene rs5929 (Exon10) T allele $(P=0.022<0.05, O R=1.529,95 \% \mathrm{CI}=1.063$ 2.198), rs2738464 (Exon18) G allele $(P=0.016<0.05, O R=1.572,95$ $\% \mathrm{CI}=1.088-2.272)$ and $\mathrm{rs} 1433099$ (Exon18) $\mathrm{T}$ allele $(P=0.015<0.05$, $O R=1.578,95 \% \mathrm{CI}=1.090-2.283)$ were significantly higher than control group.

Table 3 shows the results of 3 SNPs associated with SD between SD group and control group in three gene models. LDLR gene rs5929 T allele in a dominant genetic model was associated with susceptibility to $\mathrm{SD}(\mathrm{P}=0.031<0.05), \mathrm{rs} 2738464 \mathrm{G}$ allele in an additive genetic model was associated with susceptibility to SD $(\mathrm{P}=0.007<0.05)$, rs 1433099 $\mathrm{T}$ allele in a dominant model was associated with susceptibility to SD. $(\mathrm{P}=0.023<0.05)$.

Table 1. The comparison of serum lipid levels between the patients and the controls.

\begin{tabular}{|l|c|c|c|c|}
\hline & Normal value & Case (n=139) & Control (n=139) & p value \\
\hline TG $(\mathrm{mmol} / \mathrm{L})$ & $0.5-1.7$ & $1.451 \pm 0.890$ & $1.259 \pm 0.410$ & 2.311 \\
\hline TC $(\mathrm{mmol} / \mathrm{L})$ & $3.3-5.8$ & $4.923 \pm 1.118$ & $4.412 \pm 0.741$ & 4.479 \\
\hline HDL-C $(\mathrm{mmol} / \mathrm{L})$ & $0.8-1.8$ & $1.302 \pm 0.331$ & $1.331 \pm 0.219$ & $-0.022^{*}$ \\
\hline LDL-C $(\mathrm{mmol} / \mathrm{L})$ & $2.3-3.3$ & $3.069 \pm 0.858$ & $2.794 \pm 0.668$ & 2.988 \\
\hline ApoA1 $(\mathrm{g} / \mathrm{L})$ & $1.00-1.70$ & $1.422 \pm 0.266$ & $1.432 \pm 0.208$ & -0.388 \\
\hline ApoB $(\mathrm{g} / \mathrm{L})$ & $0.60-1.10$ & $0.723 \pm 0.175$ & $0.703 \pm 0.175$ & $0.003 * *$ \\
\hline
\end{tabular}

TG: triglycerides; TC: total cholesterol; HDL-C: high-density lipoprotein cholesterol; LDL-C: low-density lipoprotein cholesterol; ApoA1: apolipoprotein A1; ApoB: apolipoprotein B; *P $<0.05, * * \mathrm{P}<0.01$ was considered statistically significant.

Table 2. The results of association of $L D L R$ gene SNP genotype and allele gene frequencies with SSHL between SSHL group and control group. Pa represented Hardy-Weinberg equilibrium in control group, $\mathrm{P}^{\mathrm{b}}$ represented the difference of genotype frequency, $\mathrm{P}^{\mathrm{c}}$ represented the difference of the allele gene frequency, using Bonferroni-Holm correction, $* \mathrm{P}<0.05, * * \mathrm{P}<0.01$ was considered statistically significant.

\begin{tabular}{|c|c|c|c|c|c|c|c|c|c|c|c|}
\hline Number & SNP & Group & & lotype (n, & & Alle & $, \%)$ & $P^{a}$ & $P^{\mathbf{b}}$ & $P^{c}$ & $\mathrm{OR}^{\mathrm{d}}, \mathbf{9 5} \% \mathrm{CI}^{\mathrm{d}}$ \\
\hline \multirow{3}{*}{1} & \multirow{3}{*}{ rs5929 } & & TT & $\mathrm{CT}$ & $\mathrm{CC}$ & $\mathrm{T}$ & $\mathrm{C}$ & \multirow{3}{*}{0.346} & \multirow{3}{*}{0.081} & \multirow{3}{*}{$0.022 *$} & \\
\hline & & SSHL & $19(13.7)$ & $60(43.2)$ & $60(43.2)$ & $98(35.3)$ & $180(64.7)$ & & & & 1.529 \\
\hline & & Control & $12(8.6)$ & $49(35.3)$ & $78(56.1)$ & $73(26.3)$ & $205(73.7)$ & & & & $1.063-2.198$ \\
\hline \multirow{3}{*}{2} & \multirow{3}{*}{ rs2738464 } & & GG & CG & $\mathrm{CC}$ & G & $\mathrm{C}$ & \multirow{3}{*}{0.276} & \multirow{3}{*}{$0.007 * *$} & \multirow{3}{*}{$0.016^{*}$} & \\
\hline & & SSHL & $15(10.8)$ & $65(46.8)$ & $59(42.4)$ & $95(34.2)$ & $183(65.8)$ & & & & 1.572 \\
\hline & & Control & $14(10.1)$ & $41(29.5)$ & $84(60.4)$ & $69(24.8)$ & $209(75.2)$ & & & & $1.088-2.272$ \\
\hline \multirow{3}{*}{3} & \multirow{3}{*}{ rs1433099 } & & TT & $\mathrm{CT}$ & $\mathrm{CC}$ & $\mathrm{T}$ & $\mathrm{C}$ & \multirow{3}{*}{0.381} & \multirow{3}{*}{0.059} & \multirow{3}{*}{$0.015^{*}$} & \\
\hline & & SSHL & $17(12.2)$ & $60(43.2)$ & $62(44.6)$ & $94(33.8)$ & $184(66.2)$ & & & & 1.578 \\
\hline & & Control & $10(7.2)$ & $48(34.5)$ & $81(58.3)$ & $68(24.5)$ & $210(75.5)$ & & & & $1.090-2.283$ \\
\hline
\end{tabular}




\section{The comparison of serum lipid parameters in patients with different genotypes}

Tables 4-6 shows serum lipid parameters were compared in 139 patients with different genotypes of rs2738464, rs1433099 and rs5929. We found there was no statistical difference in serum lipid levels of SD patients with different genotypes.

\section{Statistical analyses}

Serum lipid parameters were analysed using independent $t$ tests, and the results are presented as means \pm SD (Standard Deviation). GENEPOP v4.0 software was used to genotype Hardy-Weinberg test for SD group and control group; The software SPSS18.0 computed allele and genotype frequency for the two groups. Pearson's chi-square test indicated different alleles and genotype frequency distribution between the two groups as well as the association of alleles with susceptibility to SD among different gene models. Nonlinear logistic regression analysis was used to calculate the OR (odds ratios) and $95 \% \mathrm{CI}$ (95\% confidence intervals); All data with $\mathrm{P}<0.05$ was considered statistically significant.

\section{Discussion and conclusion}

SD features have similarities to acute ischemic diseases especially in treatment and principles. This is because features in both cases are usually sudden onset and have rapid progression. Moreover, better prognosis of treatment in the acute phase and healing of SD also resembles ischemic disease. Some scholars believe that lipid metabolism disorders are risk factors for SD and coronary heart disease [7]. The pathogenesis of vascular risk factors was also similar: for example, coronary artery embolization is the main artery embolism affecting myocardial blood supply, and SD is microvascular embolism, affecting the inner ear blood supply [7]. Tagaya reported that patients with SD were similar with acute ischemic events in their magnetic resonance imaging, such as myocardial infarction and cerebral vascular accident [8]. Chien found that phosphodiesterase 4D gene polymorphism was not only associated with ischemic stroke, but also increased women's susceptibility to SD [9]. Numerous studies confirmed that polymorphisms within IL-6, methylenetetrahydrofolate reductase, prothrombin, and Factor $\mathrm{V}$ Leiden genes are related to coronary heart disease and SD. To a certain extent, these gene polymorphisms may also determine the susceptibility to SD.

Table 3. The results of association of $L D L R$ gene SNPs with SSHL under three gene models. $\mathrm{P}^{\mathrm{d}}$ represented the difference of allele gene frequency in dominant model, $\mathrm{P}^{\mathrm{e}}$ represented the difference of allele gene frequency in additive model, $\mathrm{P}^{\mathrm{f}}$ represented the difference of allele gene frequency in recessive model, using Bonferroni-Holm correction, $* \mathrm{P}<0.05, * * \mathrm{P}<0.01$ represented the differences were statistically significant.

\begin{tabular}{|c|c|c|c|c|c|c|c|c|}
\hline Number & SNP & Group & & enotype (n & & $\mathbf{P}^{\mathbf{d}}$ & $\mathbf{P}^{\mathbf{e}}$ & $\mathbf{P}^{\mathrm{f}}$ \\
\hline \multirow{3}{*}{1} & \multirow{3}{*}{ rs5929 } & & $\mathrm{TT}$ & $\mathrm{CT}$ & $\mathrm{CC}$ & \multirow{3}{*}{$0.031 *$} & \multirow{3}{*}{0.081} & \multirow{3}{*}{0.182} \\
\hline & & SSHL & 19(13.7) & $60(43.2)$ & $60(43.2)$ & & & \\
\hline & & Control & $12(8.6)$ & $49(35.3)$ & $78(56.1)$ & & & \\
\hline \multirow{3}{*}{2} & \multirow{3}{*}{ rs2738464 } & & GG & $\mathrm{CG}$ & $\mathrm{CC}$ & \multirow{3}{*}{$0.003 *$} & \multirow{3}{*}{$0.007^{*}$} & \multirow{3}{*}{0.844} \\
\hline & & SSHL & $15(10.8)$ & $65(46.8)$ & $59(42.4)$ & & & \\
\hline & & Control & $14(10.1)$ & $41(29.5)$ & $84(60.4)$ & & & \\
\hline \multirow{3}{*}{3} & \multirow{3}{*}{ rs1433099 } & & $\mathrm{TT}$ & $\mathrm{CT}$ & $\mathrm{CC}$ & \multirow{3}{*}{$0.023 *$} & \multirow{3}{*}{0.059} & \multirow{3}{*}{0.156} \\
\hline & & SSHL & $17(12.2)$ & $60(43.2)$ & $62(44.6)$ & & & \\
\hline & & Control & $10(7.2)$ & $48(34.5)$ & $81(58.3)$ & & & \\
\hline
\end{tabular}

Table 4. The comparison of serum lipid parameters in patients with different genotype of rs5929. $\mathrm{P}<0.05$ was considered statistically significant.

\begin{tabular}{|c|c|c|c|c|c|c|}
\hline & CC & n & CT & n & TT & P \\
\hline TG & $1.499 \pm 0.903$ & 60 & $1.438 \pm 0.933$ & 60 & $1.338 \pm 0.724$ & 0.785 \\
\hline TC & $5.026 \pm 1.152$ & 60 & $4.908 \pm 1.150$ & 60 & $4.637 \pm 0.880$ & 19 \\
\hline HDL-C & $1.27(1.033-1.513)$ & 60 & $1.305(1.038-1.51)$ & 60 & $1.29(1.1-1.47)$ & 19 \\
\hline LDL-C & $3.202 \pm 0.933$ & 60 & $2.989 \pm 0.806$ & 60 & $2.906 \pm 0.743$ & 19 \\
\hline APOA1 & $1.399 \pm 0.277$ & 60 & $1.43 \pm 0.269$ & 60 & $1.469 \pm 0.227$ & 0.881 \\
\hline APOB & $0.705(0.58-0.84)$ & 60 & $0.665(0.61-0.8)$ & 60 & $0.71(0.58-0.83)$ & 19 \\
\hline
\end{tabular}

Table 5. The comparison of serum lipid parameters in patients with different genotypes of rs 2738464 . $\mathrm{P}<0.05$ was considered statistically significant.

\begin{tabular}{|c|c|c|c|c|c|c|}
\hline & CC & n & CG & n & GG & P \\
\hline TG & $1.495 \pm 0.893$ & 59 & $1.249 \pm 0.935$ & 65 & $1.249 \pm 0.935$ & 15 \\
\hline TC & $4.887 \pm 1.064$ & 59 & $4.835 \pm 1.005$ & 65 & $4.835 \pm 1.005$ & 15 \\
\hline HDL-C & $1.301 \pm 0.325$ & 59 & $1.405 \pm 0.315$ & 65 & $1.405 \pm 0.315$ & 0.633 \\
\hline LDL-C & $3.064 \pm 0.880$ & 59 & $2.871 \pm 0.759$ & 65 & $2.871 \pm 0.759$ & 15 \\
\hline APOA1 & $1.404 \pm 0.244$ & 59 & $1.56 \pm 0.322$ & 65 & $1.56 \pm 0.322$ & 0.421 \\
\hline APOB & $0.67(0.6-0.85)$ & 59 & $0.68(0.585-0.8)$ & 65 & $0.76 \square 0.65-0.81 \square$ & 15 \\
\hline
\end{tabular}

Table 6. The comparison of serum lipid parameters in patients with different genotypes of rs 1433099 . $\mathrm{P}<0.05$ was considered statistically significant.

\begin{tabular}{|c|c|c|c|c|c|c|c|}
\hline & $\mathrm{CC}$ & $\mathbf{n}$ & CT & $\mathbf{n}$ & TT & $\mathbf{n}$ & $\mathbf{P}$ \\
\hline TG & $1.540 \pm 0.945$ & 62 & $1.406 \pm 0.807$ & 60 & $1.281 \pm 0.974$ & 17 & 0.500 \\
\hline $\mathrm{TC}$ & $4.860 \pm 1.096$ & 62 & $4.994 \pm 1.174$ & 60 & $4.894 \pm 1.041$ & 17 & 0.803 \\
\hline HDL-C & $1.275 \pm 0.323$ & 62 & $1.294 \pm 0.347$ & 60 & $1.43 \pm 0.287$ & 17 & 0.227 \\
\hline LDL-C & $3.065 \pm 0.901$ & 62 & $3.116 \pm 0.838$ & 60 & $2.921 \pm 0.795$ & 17 & 0.712 \\
\hline APOA1 & $1.388 \pm 0.235$ & 62 & $1.418 \pm 0.274$ & 60 & $1.562 \pm 0.315$ & 17 & 0.055 \\
\hline APOB & $0.65(0.578-0.82)$ & 62 & $0.69(0.61-0.8)$ & 60 & $0.78(0.655-0.845)$ & 17 & 0.314 \\
\hline
\end{tabular}


$L D L R$ as a candidate gene for coronary heart disease has been studied extensively. As a result, in this study we choose $L D L R$ as a candidate gene for research of susceptibility to SD. $L D L R$ gene is located on the short arm of human chromosome 19 terminal (ie 19p13.3) and is composed of 18 exons and 17 introns, a total length of $45 \mathrm{~Kb}$. $L D L R$ polymorphisms are thought to affect the activity of the LDLR protein by altering its structure and subsequent function [10]. LDLR plays an important role in lipid metabolism and can be identified and combined with cholesterol specifically via apolipoprotein $\mathrm{E}$ and apolipoprotein $\mathrm{B}_{100}$, which decompose into bile acids, steroid, and vitamin D3 after being swallowed. $2 / 3$ of normal human plasma low-density lipoprotein (LDL) is degraded in the LDL-R pathway, which can clear $65 \%-70 \%$ of LDL. Under long-term high cholesterol, macrophages are induced to swallow excess LDL and absorb the LDL cholesterol, causing cholesterol to be deposited in the phagocytic cells and become "foam" cells [11]. Low density lipoprotein of oxidative modification (OX-LDL) and cholesterol will affect the structure and function of artery intima. Some studies have shown that $L D L R$ polymorphisms play a key role in regulating body's cholesterol and triglycerides (TG) concentrations [12]. Through many articles and reviews, Ashavaid found that $L D L R$ gene polymorphism is closely related to lipid metabolism which has a strong correlation to early atherosclerosis plaque formation [13]. The blood supply of the inner ear is from the anterior inferior cerebellar artery, which is a terminal artery and has no collateral circulation. If some changes in blood lipid factors occur in the inner ear, dysfunction in microcirculation of cochlea and vestibular can easily occur. Blood lipid metabolism is closely linked to blood rheology and vascular disease. Lipids attached to the surface of the red blood cells and platelets, can reduce the ability of red blood cells to carry charges, enhancing the viscosity between cells and increase blood viscosity consequently slowing blood flow. An increase in cholesterol in the blood cell membrane components promotes cell sclerosis, weakens its ability to carry oxygen, and causes deformation, leading to hypoxia injury of capillary endothelial cell in inner ear. Triglyceride metabolites can damage the vascular endothelial cells directly, promote microthrombosis, reduce blood supply in the inner ear, and result in the onset of SD [14]. Chang et al. [15], found that SD of subjects with high cholesterol was 1.62 times than normal lipid objects, Cox proportional risk-adjusted return ratio of $1.6(95 \% \mathrm{CI}=1.39-1.85)$ by a retrospective cohort study. As a result, high cholesterol was considered a risk factor for the onset of SD and focus on hearing changes of objects with high cholesterol in clinical practice. X. Zhang found that there were $20.4 \%$ SD patients with hypertension, coronary artery disease, or diabetes, and $49.6 \%$ patients with hyperlipidemia [16]. When Chinchillas were fed a high cholesterol diet, visible lipid deposits in the edge layer of cochlear stria and outer hair cell histology, suggesting that high cholesterol can reduce cochlear blood vessels and cause hearing loss [17]. It was shown that lipid fluidity and sturdiness of the outer hair cells in the cochlea membrane are associated with cochlear potential amplification [18]. High cholesterol not only affects the blood supply to the inner ear, but also destroys the structure of outer hair cell, as result of affecting its activity.

This study is the first large-sample case-control association analysis study about the relationship between $L D L R$ gene polymorphism with SD. SNP genotyping methods chose fluorescent multiplex ligase reaction (iMLDR) technology to ensure high accuracy and detection efficiency. It established dominance, additive, and recessive genetic models to analyze the association between the $L D L R$ gene polymorphisms and $\mathrm{SD}$, thus reducing false positive rates in case and control groups of genotype and allele frequencies results. By evaluating the relationship of 3 SNPs loci of $L D L R$ gene and SD, the study found the frequencies of
LDLR gene rs5929 T allele, rs2738464 locus G allele, rs1433099 T allele of SD group were significantly higher in the control group, and nonconditional logistic regression analysis showed that $L D L R$ gene rs5929 $\mathrm{T}$ allele, rs2738464 locus $\mathrm{G}$ allele, rs $1433099 \mathrm{~T}$ allele may be risk factor for onset of SD. Scholars have found that the rs1433099 polymorphism associated with cerebral infarction and $\mathrm{T}$ allele of this locus may increase the risk of cerebral infarction [19]. Our study is the first to discover the correlation between this site and SD and reflect the similar pathogenesis of SD and acute ischemic disease; rs5929 located in 13th exon of $L D L R$ gene. By consideration of the relation of $L D L R$ gene structure and function, the 7-14th exon is the second protein domain of encoding LDLR, EGF precursor homology domain, and it plays an important role in the fixed membrane protein, receptor binding ligand, the solution receptor of ligands in the lysosome, receptor synthesis and recycling. This region of SNP polymorphisms may affect LDLR activity or structure and will not only result in the reduced cholesterol and LDLR binding and intracellular swallow decomposition, but also increases the level of serum total cholesterol, one risk factor of SD [20]. The rs 2738464 located in the 3'UTR region of $L D L R$ gene. It belongs to transcriptional regulation which affects the stability of LDLR expression and protein synthesis structure, thereby affecting LDL via LDLR degradation pathway. Excessive plasma LDL was modified by oxidation to form OX-LDL. OX-LDL could cause sudden hearing loss by affecting the structure and function of the arterial intima and forming thrombosis in the microcirculation [21]. These positive SNPs loci are different from the existing positive SNPs loci of the $L D L R$ gene which are associated with coronary heart disease, due to different pathogenesis of two diseases and the selection of the target population, or other factors. Therefore, individuals carrying these three alleles may be high-risk groups of SD. In this study, no association is found between serum lipid levels and different genotypes in rs5929, rs2738464 and rs1433099 loci for SD patients. It may be caused by the limited sample number and the sample imbalance on gender and age. In the future research, we need to increase the sample number and then explore the correlation between LDLR genotype and blood lipid level.

In this study, all subjects were from Han nationality, Shaanxi population, only representative of Han nationality of the northern population, indicating a need to extend the study to different regions, different ethnic groups, and the choice of a larger sample size for verification.

\section{Reference}

1. Zhang X, Xu M, Duan ML, Nicolai P (2009) Sudden Deafness clinical epidemiological analysis. Chinese J Otolo 7: 152-156.

2. Uchida Y, Sugiura S, Nakashima T, Ando F, Shimokata H (2011) Contribution of $1425 \mathrm{G} / \mathrm{A}$ polymorphism in protein kinase $\mathrm{C}$-Eta $(\mathrm{PRKCH})$ gene and brain white matter lesions to the risk of sudden sensorineural hearing loss in a Japanese nested casecontrol study. J Neurogenet. 25: 82-87. [Crossref]

3. Mohammed AA (2014) Lipid Profile among Patients with Sudden Sensorineural Hearing Loss. Indian J Otolaryngol Head Neck Surg 66: 425-428. [Crossref]

4. Lee JS, Kim DH, Lee HJ, Kim HJ, Koo JW, et al. (2015) Lipid profiles and obesity as potential risk factors of sudden sensorineural hearing loss. PLoS One 10: e122496. [Crossref]

5. Zhang L, Yuan F, Liu P, Fei Y, Huang Y, et al. (2013) Association between PCSK9 and LDLR gene polymorphisms with coronary heart disease: case-control study and metaanalysis. Clin Biochem 46: 727-732. [Crossref]

6. Deangelis AM, Roy-O'Reilly M, Rodriguez A (2014) Genetic alterations affecting cholesterol metabolism and human fertility. Biol Reprod 91: 117. [Crossref]

7. Bhanushali AA, Contractor A, Das BR (2012) Evaluation of the promoter polymorphism-911C $>\mathrm{A}$ in the 3-hydroxy-3-methylglutaryl-Coenzyme A reductase gene with coronary artery disease risk and cholesterol levels in a population from Western India. Transl Res 160: 445-446. [Crossref] 
8. Tagaya M, Teranishi M, Naganawa S, Iwata T, Yoshida T, et al. (2010) 3 Tesla magnetic resonance imaging obtained 4 hours after intravenous gadolinium injection in patients with sudden deafness. Acta Otolaryngol 130: 665-669.

9. Chen CY, Tai SY, Wang LF, His E, Chang NC, et al. (2016) Phosphodiesterase 4D gene polymorphisms in sudden sensorineural hearing loss. Eur Arch Otorhinolaryngol 273: 2403-2409. [Crosref]

10. Jamaldini SH, Babanejad M, Mozaffari R, Nikzat N, Jalalvand K, et al. (2014) Association of Polymorphisms at LDLR Locus with Coronary Artery Disease Independently from Lipid Profile. Acta Med Iran 52: 352-359. [Crossref]

11. Gao F, Ihn HE, Medina MW, Krauss RM (2013) A common polymorphism in the LDL receptor gene has multiple effects on LDL receptor function. Hum Mol Genet 22: 1424 1431. [Crossref]

12. Du J, Fang DZ, Gong RR, Tang H, Li R, et al. (2010) Effects of Pvu II polymorphism in low density lipoprotein receptor gene on changes of serum lipid ratios induced by high-carbohydrate / low-fat diet in healthy youth. Sichuan Da Xue Xue Bao Yi Xue Ban 41: 239-242, 251. [Crossref]

13. Ashavaid TF, Shalia KK, Kondkar AA, Todur SP, Nair KG, et al. (2002) Gene polymorphism and coronary risk factors in Indian population. Clin Chem Lab Med 40: 975-985. [Crossref]
14. Lu Y (2007) Clinical study of sudden deafness and microcirculation relationship. Anhui Medical University: 1-35. [Crossref]

15. Chang SL, Hsieh CC, Tseng KS, Weng SF, Lin YS (2014) Hypercholesterolemia is correlated with an increased risk of idiopathic sudden sensorineural hearing loss: a historical prospective cohort study. Ear Hear 35 : 256-261. [Crossref]

16. Zhang X, Xu X, Ma W, Zhang Q, Tong H, et al. (2015) A clinical study of sudden deafness. Acta Oto-Laryngologica 135: 1031-1035. [Crossref]

17. Gratton MA, Wright CG (1992) Alterations of inner ear morphology in experimental hypercholesterolemia. Hear Res 61: 97-105.

18. Lin HC, Wang CH, Chou YC, Shih CP, Chu YH, et al. (2015) The correlation between lipoprotein ratios and hearing outcome in idiopathic sudden sensorineural hearing loss patients. Clin Otolaryngol 40: 355-362. [Crossref]

19. Yan HC, Wang W, Dou CW, Tian FM, Qi ST (2014) Relationships of LDLR genetic polymorphisms with cerebral infarction: a meta-analysis. Mol Biol Rep 41: 4425-4434. [Crossref]

20. Vincent J (2014) Reducing Elevated Plasma LDL Cholesterol: The Central Role of the LDL Receptor. Clin Pharmacol Ther 96: 3-7. [Crossref]

21. Ye H, Zhao Q, Huang Y, Wang L, Liu H, et al. (2014) Meta-Analysis of Low Density Lipoprotein Receptor (LDLR) rs2228671 Polymorphism and Coronary Heart Disease. Biomed Res Int: 1-6. [Crossref]

Copyright: $\odot 2020$ Cui LC. This is an open-access article distributed under the terms of the Creative Commons Attribution License, which permits unrestricted use, distribution, and reproduction in any medium, provided the original author and source are credited. 